Gazi University
Pournal of Science
PART C: DESIGN AND TECHNOLOGY
http://dergipark.gov.tr/gujsc

\title{
A Comparison of Two Discrimination Spectrometers
}

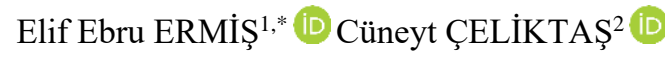 \\ ${ }^{1}$ Izmir Tinaztepe University, Vocational School of Health Services, Medical Imaging Techniques Department, 35400, Buca/IZMIR \\ ${ }^{2}$ Ege University, Faculty of Science, Physics Department,35100, Bornova/IZMIR
}

\section{Article Info}

Research article

Received: $25 / 02 / 2021$

Revision: $25 / 04 / 2021$

Accepted: $28 / 04 / 2021$

Keywords

Gamma-ray spectroscopy

${ }^{137} \mathrm{Cs}$

$\operatorname{NaI}(T l)$

Puls shape discrimination

\begin{abstract}
In order to obtain a neat gamma spectrum, two spectrometers were examined for the discrimination of the low-energy part of the ${ }^{137} \mathrm{Cs}$ gamma-ray spectrum which has electronic noise. Pulse shape discrimination (PSD) time measurement (timing) method was used for this purpose. This method, briefly, depends on the rise time measurement process of the signals. While implementing the process, a constant fraction discriminator (CFD) and a pulse shape analyzer (PSA) devices were used separately. Obtained spectra from the spectrometers were compared with each other. To perform an exact comparison, partial and complete discrimination processes were realized. After the comparison, it was concluded that the discrimination process through the spectrometer with PSA device was more successful than that of the other one. It was suggested that the PSD method using the PSA device would give a clearer gamma spectrum when accumulating it by a $\mathrm{NaI}(\mathrm{Tl})$ scintillation detector.
\end{abstract}

\section{INTRODUCTION}

Energy spectrum of a radioactive source gives us the information about the source showing the spectral distribution of energy. In other words, energy spectrum of a radioactive source is the number of the particles in each energy value for the detected energy interval.

The evaluation of the interaction in a medium of the particles emitted from radioisotopes can be discussed as energy spectrum. Neat energy spectrum, i.e. only the energy spectrum of the particles emitted from a radioactive source (discriminated from any other artificial pulses such as background) is the main characteristic in the energy spectrum.

Distinguishing the different types of particles is known as pulse shape discrimination (PSD). With a certain scintillator, it is possible to distinguish between different types of particles by analyzing the shape of the generated light in the scintillator. This is due to the excitation of different specific energy loss of the particles $(\mathrm{dE} / \mathrm{dx})$ in the scintillator material.

In order to discriminate the unwanted signals from the original signals, a widely used method is PSD method. With this method, pulse shape differences of the different types of particles can be measured [1]. By this way, the elimination of the low-energy part of the source energy spectrum was performed in the present study.

PSD is a kind of time discrimination method. In this method, the suppression of the unwanted signals is performed by measuring the decay time of each pulse electronically, and free from the amplitude. In practice, but, only over a limited range of amplitudes can be distinguished efficiently [2]. A capacitor with a long-time constant, in a conventional preamplifier, integrates the charge produced by the scintillator light in a photomultiplier tube (PMT). Owing to the different scintillator decay times, formed signals in the PMT have different rise times (the time between $10 \%$ and $90 \%$ of the amplitude in the 
leading edge of a signal) naturally, and these rise time differences can be on the order of a microsecond [3]. Further information about PSD is available at numerous works [2, 4-6]. For example, PSD technique was used to discern between neutron and gamma-ray interactions in certain organic scintillators by Ruch et al. [7]. Yanagida et al. [8], Doucet et al. [9] and Senoville et al. [10] used the PSD method for neutrongamma discrimination. Recker et al. [11] utilized from the PSD technique in various electronics for particle detection. This technique was used to discriminate the photons and neutrons in the energy range of $0.1-2 \mathrm{GeV}$ by Sugiyama et al. [12]. It is clear that the PSD is a daily method in discriminating the different particles. The method was also used here with respect to this.

Two spectrometers were used in this study to discriminate the low-energy part of the ${ }^{137} \mathrm{Cs}$ gamma-ray spectrum through PSD method. Partial and complete discriminations were followed to compare their performances for the elimination of unwanted parts of the spectrum.

\section{MATERIALS AND METHODS}

When the particles with different types and energies interact with detector material (scintillator), they generate the signals with different magnitudes and rise times in the detector. Thus, the rise times of the signals generated by the gamma particles will be different from the noise or unwanted signals. By following and subjecting the gamma signals to timing process, timing signals are produced. These timing signals are then gated with the detector signals coincidentally in the MCA. In this way, it is possible to record the triggered signals by the timing signals. Finally, the discriminated neat source spectrum can be recorded. This process can be called as PSD technique. This technique will be followed in the present study.

Block diagrams of the spectrometer set-ups for the PSD application are illustrated in Figs. 1(a) and 1(b). Ortec 401A BIN, Bicron 3x3 inch NaI(Tl) inorganic scintillation detector, Ortec 266 photomultiplier base (PMT), Ortec 459 power supply (PS), Ortec 113 preamplifier (PA), Ortec 451 spectroscopy amplifier (AMP), Ortec 427A Delay amplifier (DA), Ortec 420A timing single channel analyzer (SCA), Ortec 463 constant fraction discriminator (CFD), Ortec 416A gate and delay generator (GDG), Ortec 552 pulse shape analyzer (PSA), Ortec 566 time to amplitude converter (TAC) and Ortec Trump 8K multichannel analyzer (MCA) card mounted in a computer for data collection were used in the spectrometers. NaI detector has an aluminum window of $0.5 \mathrm{~mm}$ to protect it from artificial counts arising from the ambient light. 

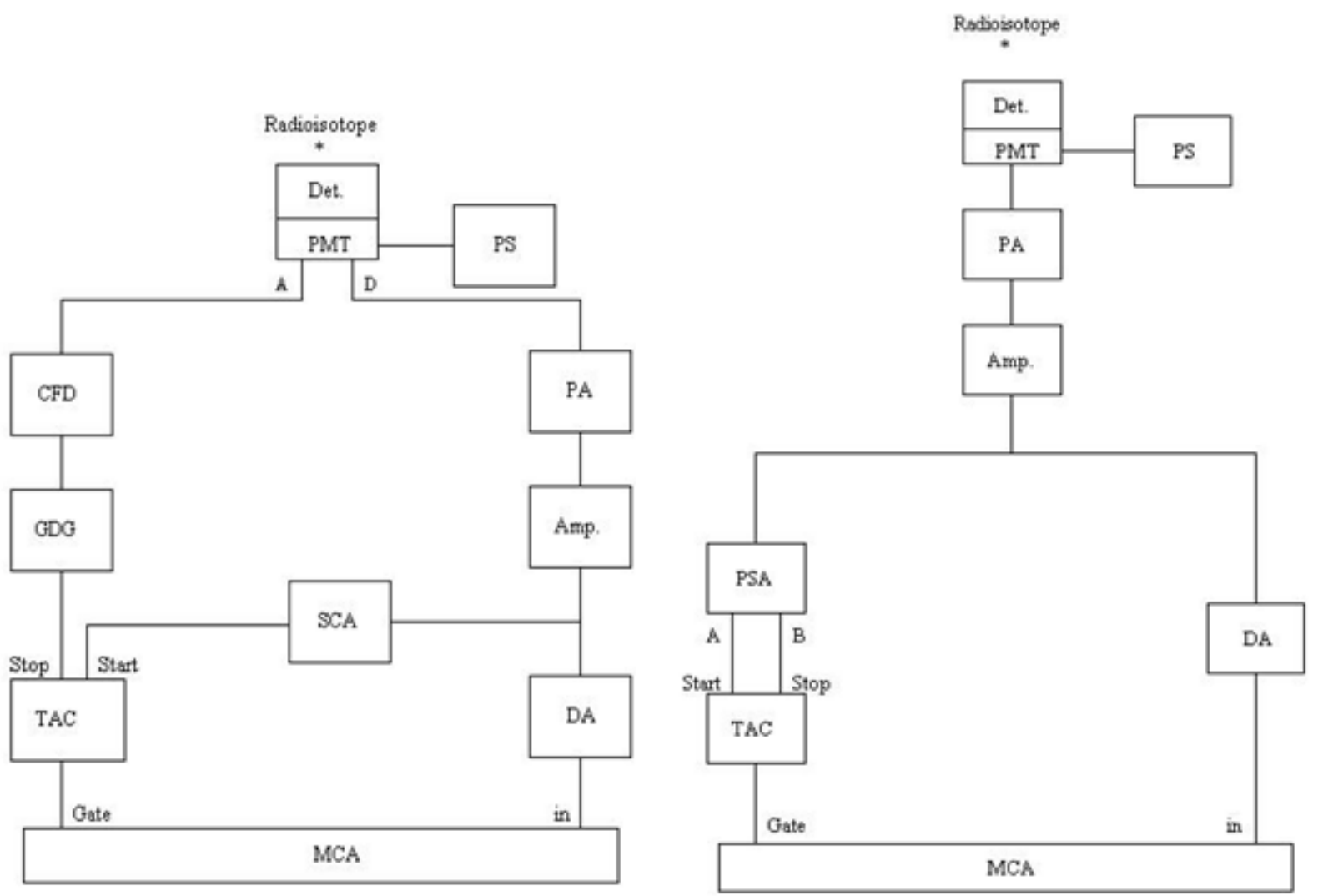

Figure1.Block diagrams of (a) first set-up, (b) second set-up. (Det.: Detector, PMT: Photomultiplier tube, PS: Power supply, PA: Preamplifier, Amp.: Amplifier, DA: Delay amplifier, SCA: Timing single channel analyzer, CFD: Constant fraction discriminator, GDG: Gate and delay generator, PSA: Pulse shape analyzer, TAC: Time to amplitude converter, MCA: Multichannel analyzer).

${ }^{137} \mathrm{Cs}$ radiation source $(<5 \mu \mathrm{Ci})$, which the radioactive material is embedded in a solid mylar disk of diameter $25 \mathrm{~mm}$ with an active area of $5 \mathrm{~mm} 2$ and an overall thickness of $5 \mathrm{~mm}$, was placed at $5 \mathrm{~cm}$ from the detector surface. Passive background reduction was performed by shielding the detector with lead blocks. The spectrometers were calibrated according to the photopeak energy of $662 \mathrm{keV}$ of the source.

In the spectrometers, the energy output of the PA is shaped by the main amplifier and sent to the MCA through the DA. The signal shapes from the DA for both spectrometers are shown in figures 2(a) and 3(a). 

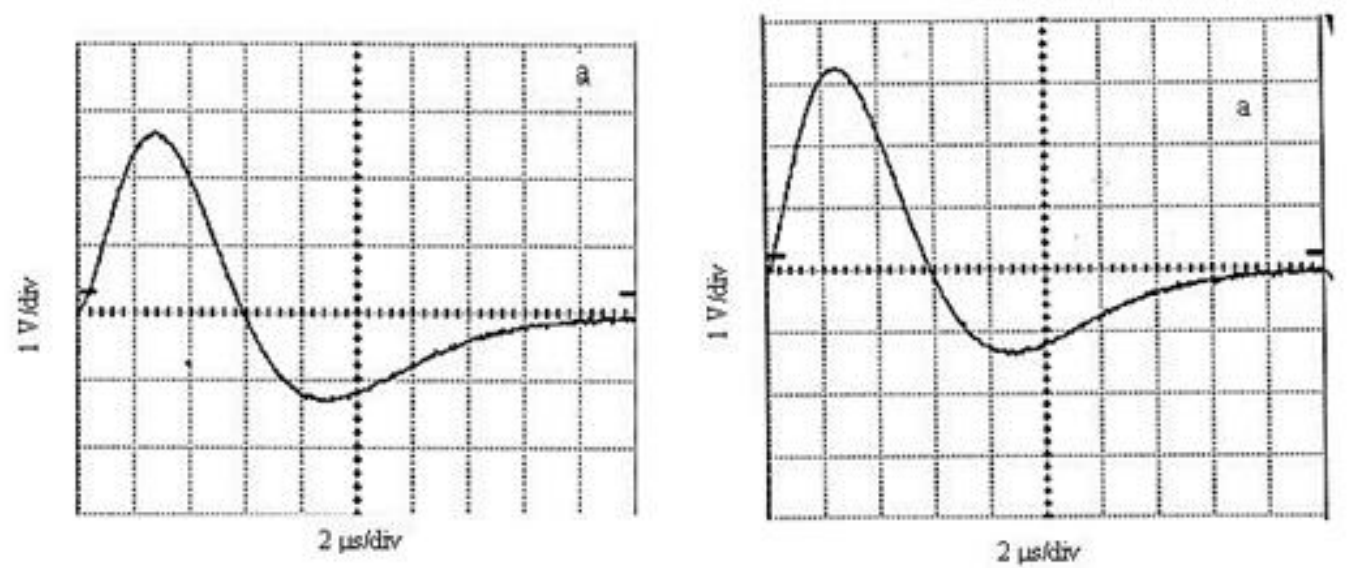

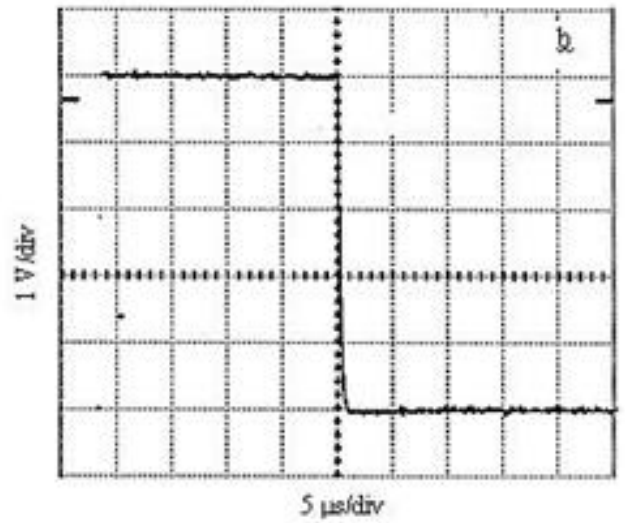

Figure 2

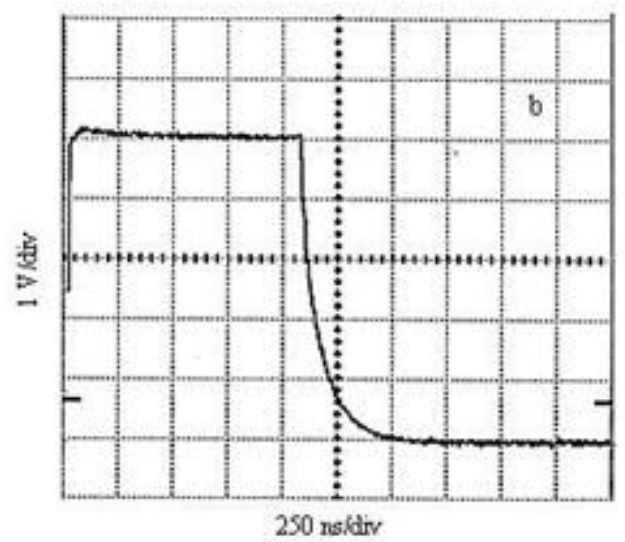

Figure 3

Figure 2. Signal shapes from the first set-up: a) DA output, b) TAC output.

Figure 3. Signal shapes from the second set-up: a) DA output, b) TAC output.

The time signal of the PMT was used in the left part of the first set-up in figure 1(a). The start signal of the TAC is generated by the SCA connected to the main amplifier, and the stop signal is produced by the CFD connected to GDG. A logic signal as in figure 2(b) is generated by the TAC corresponding to the time difference between the start and the stop signals. This logic signal gates the MCA and thus the lowenergy part of the energy spectrum is discriminated.

In the second set-up, the PSA produces the start and the stop signals corresponding to the leading edge time interval (10\%-90\%) of the output of the main amplifier. The time difference between these signals creates a logic output on the TAC and the output gates the MCA. The output of the TAC is shown in figure 3(b). By setting the TAC window, the MCA will not record the low-energy part of the ${ }^{137} \mathrm{Cs}$ energy spectrum.

As can be seen in the circuit schemes of the spectrometers above, anode and dynode outputs of the PMT were used in the first setup. Its only dynode output was utilized in the second setup also. The dynode output of the PMT generates the slow signals (long leading edge time), in addition, anode output produces the fast signals (short leading edge time). First spectrometer depends on the fast-slow coincidence signal recording process. In the second setup, PSD technique was applied only on the slow signals. This is the main difference between the used setups. The results from the setups are given in the next section. 


\section{RESULTS AND DISCUSSION}

The performances of the spectrometers were tested experimentally on the energy spectrum of ${ }^{137}$ Cs. Gamma ray energy spectrum was collected in the MCA card where it is divided into 2048 channels and then stored in a computer.

The gamma ray energy spectra of ${ }^{137} \mathrm{Cs}$ source from the first and the second spectrometers with a $300 \mathrm{~s}$ run time at room temperature of $298 \mathrm{~K}$ can be seen in figures 4 and 7. In addition, partial and complete discriminated energy spectra for both set-ups are presented in figures 5, 6, 8 and 9 .

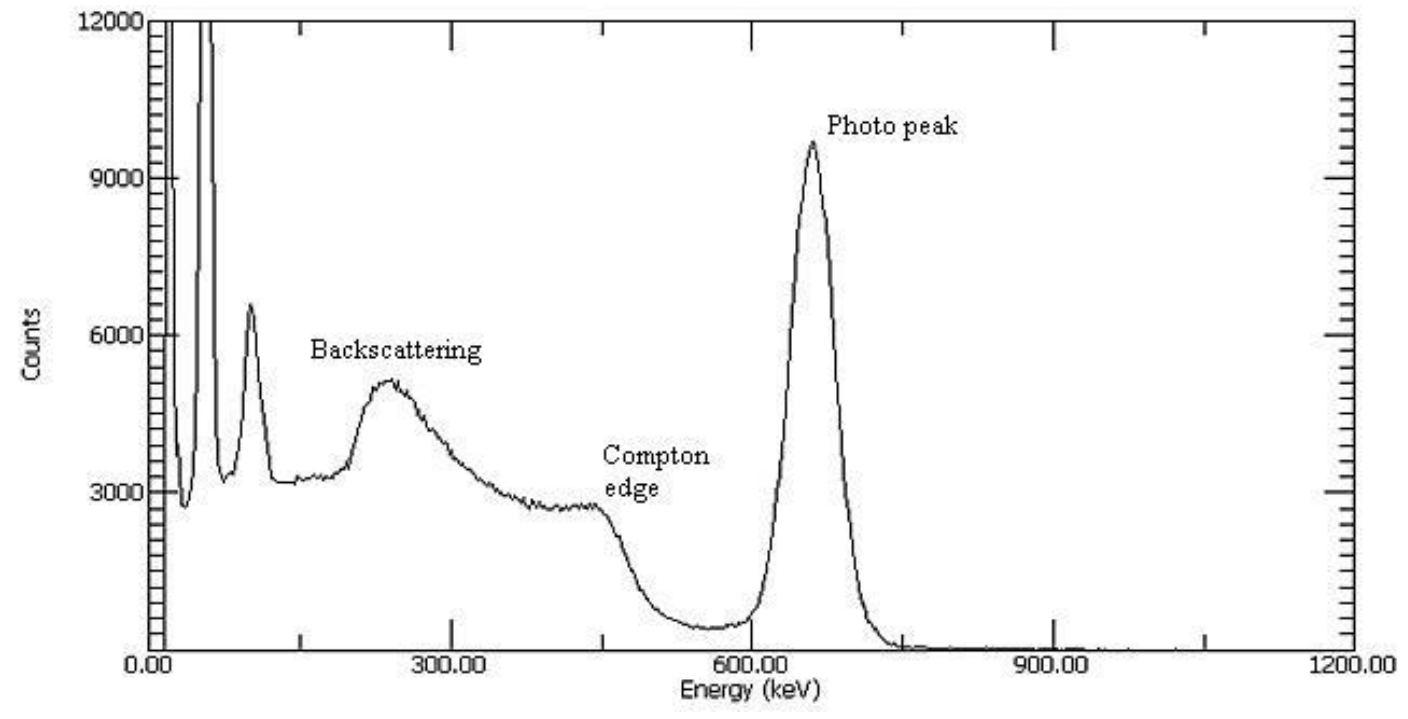

Figure 4.Direct spectrum of ${ }^{137}$ Cs from the first set-up.

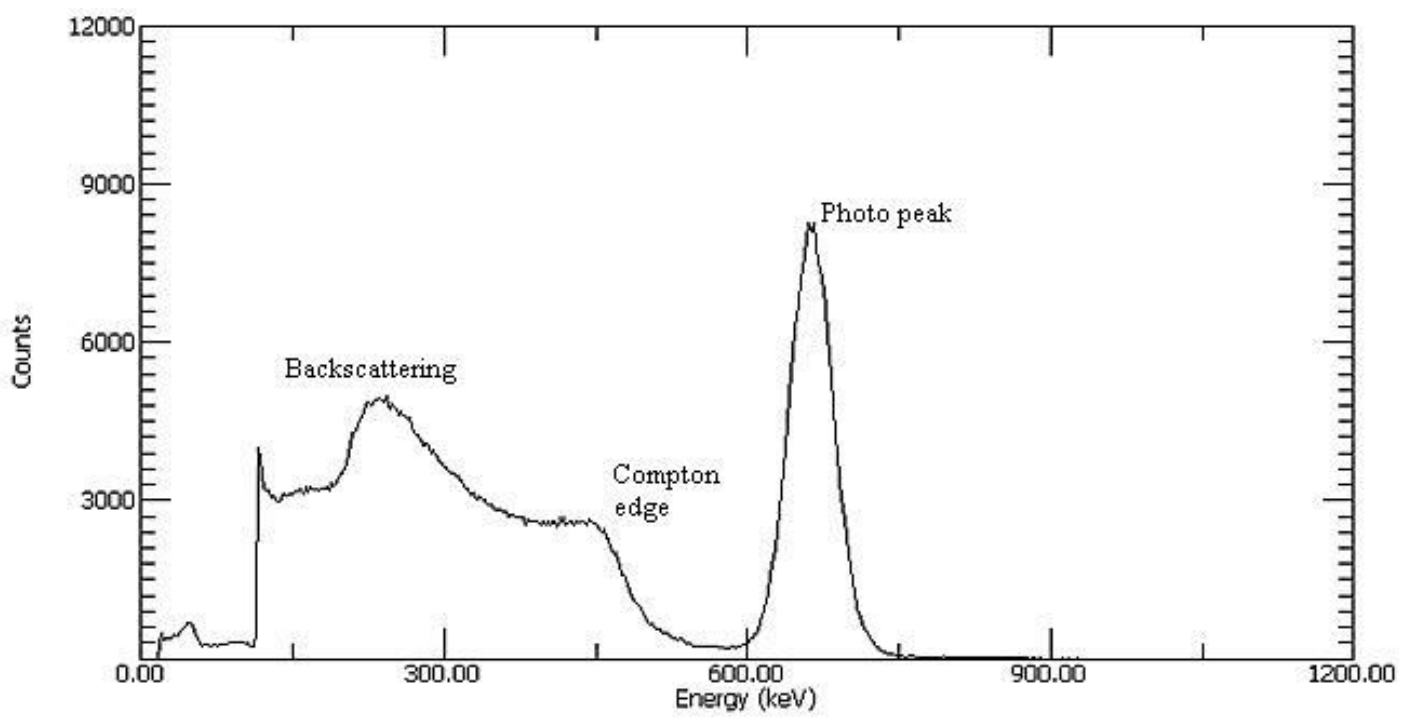

Figure 5.Partial discriminated energy spectrum of ${ }^{137}$ Cs from the first set-up. 


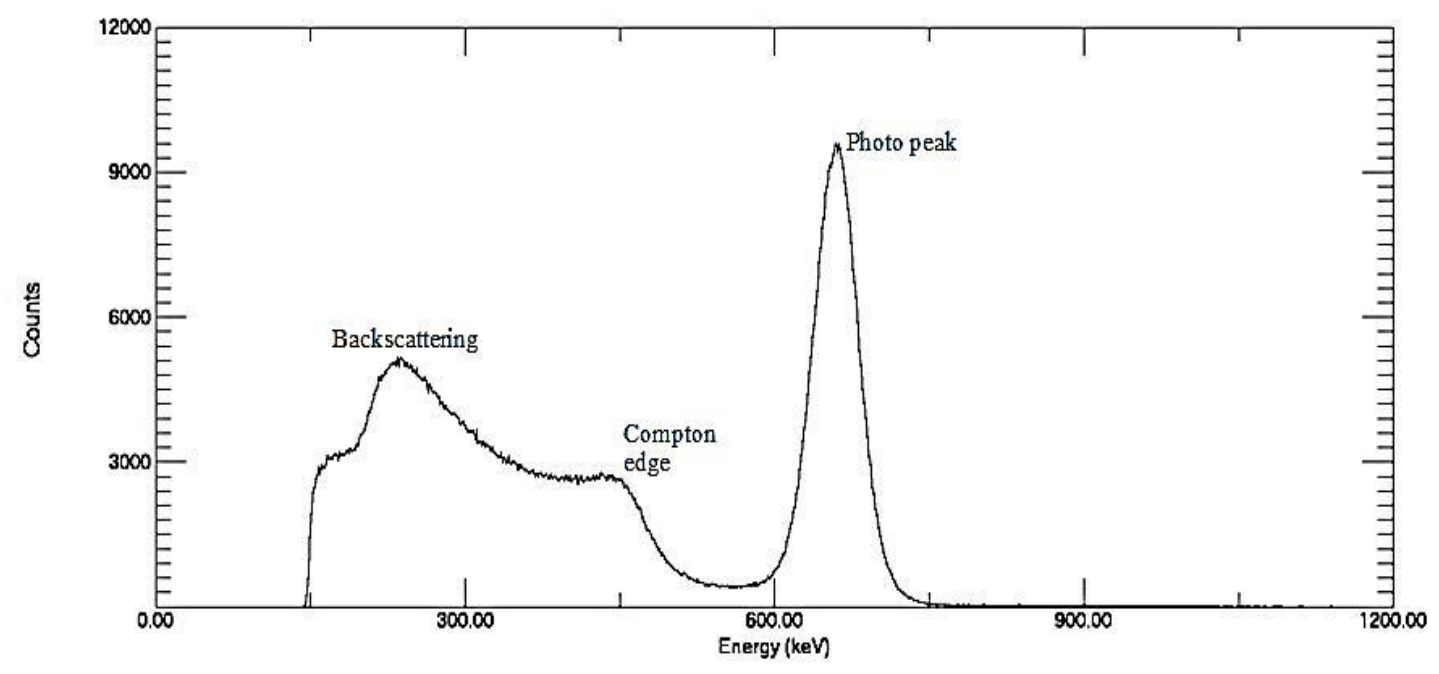

Figure 6. Complete discriminated energy spectrum of ${ }^{137}$ Cs from the first set-up.

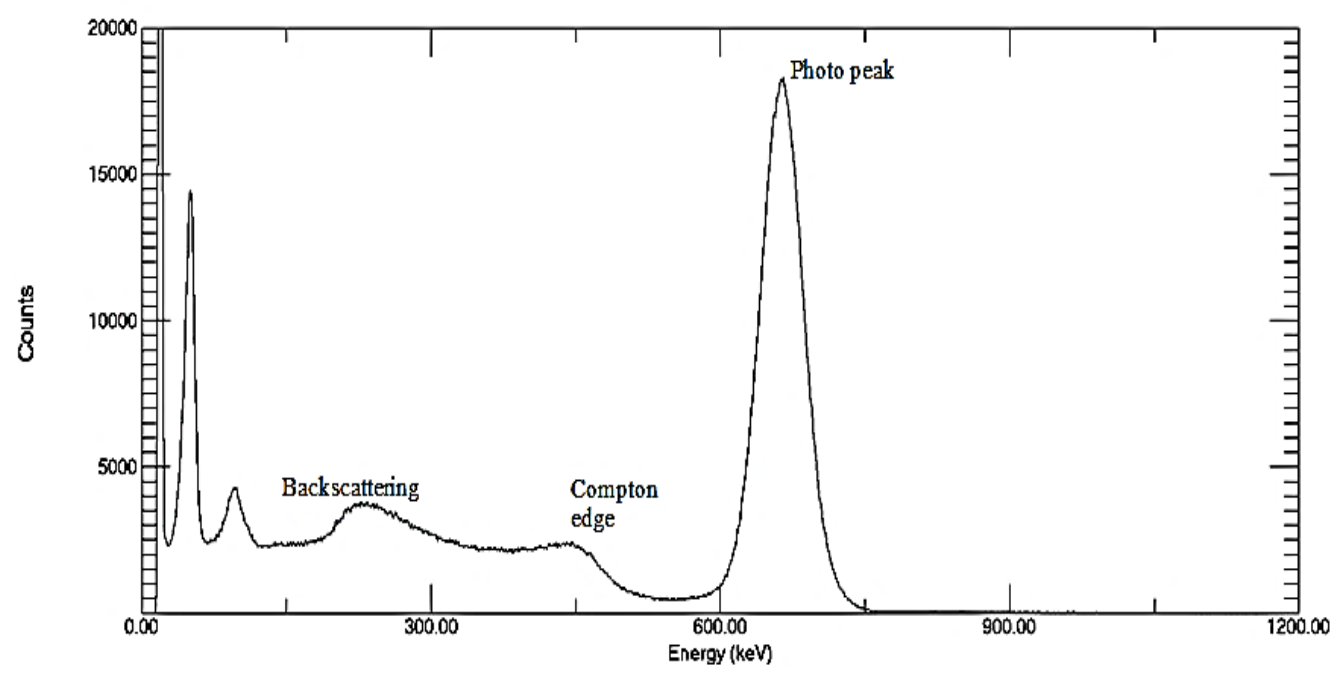

Figure 7.Direct spectrum of ${ }^{137}$ Cs from the second set-up.

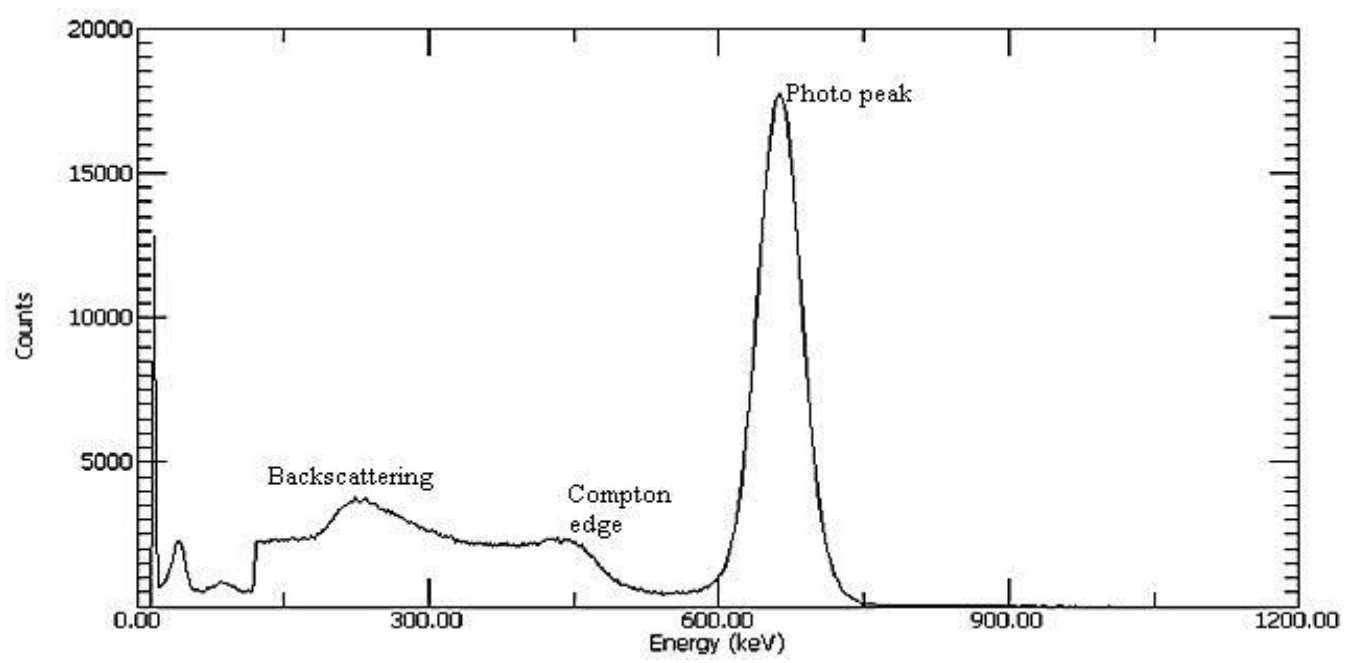

Figure 8.Partial discriminated energy spectrum of ${ }^{137}$ Cs from the second set-up 


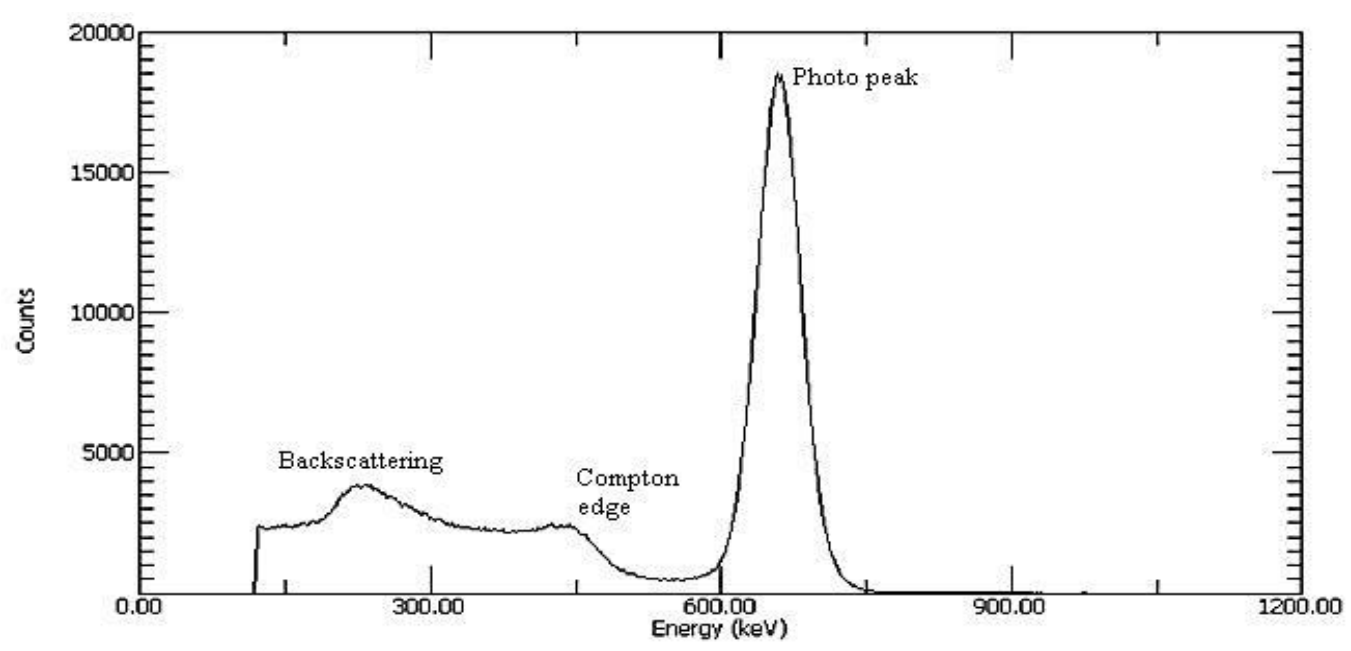

Figure 9. Complete discriminated energy spectrum of ${ }^{137}$ Cs from the second set-up.

The detector dead time was between $18 \%$ and $20 \%$, and the obtained background spectrum was given in figure 10. The background spectrum has been subtracted from all the energy spectra given in Figures 4 to 9.

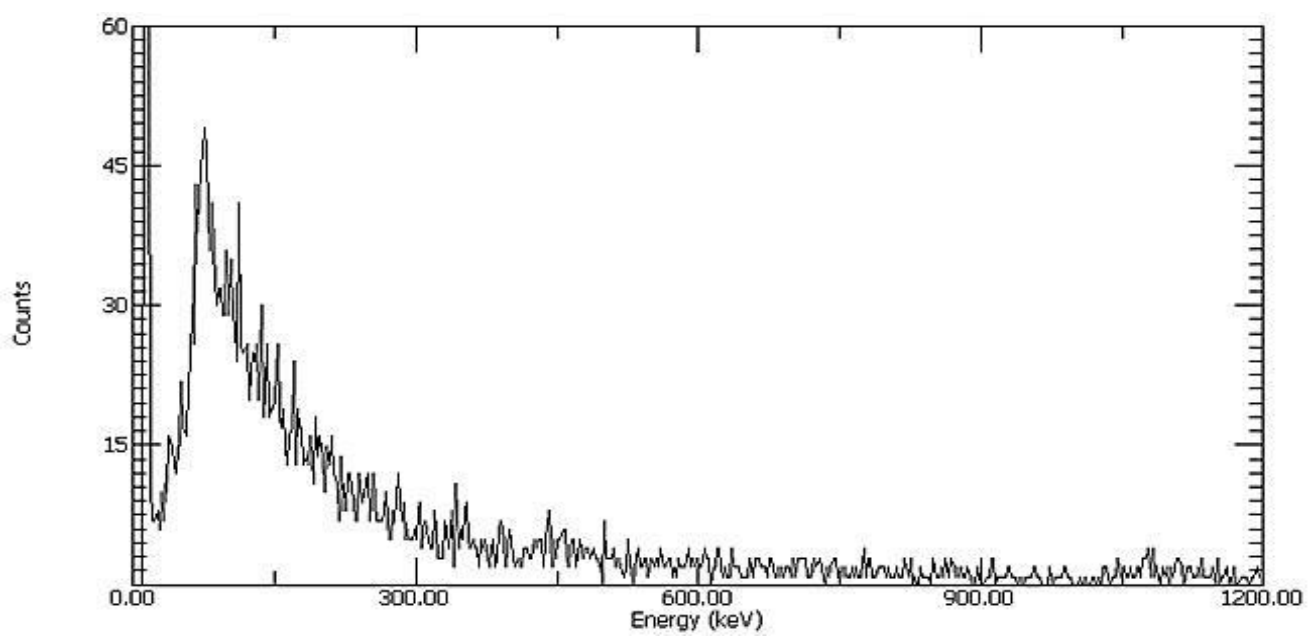

Figure 10.Detector background spectrum.

The energy resolutions of both spectrometers were calculated to check the system performance. The energy resolutions for the photo peaks in figures 6 and 9 were found to be $7.55 \%$ and $7.53 \%$, respectively. It can be said from the obtained results above that the followed PSD method, which is used for the discrimination of neutron and gamma particles in literature, was successful in the discrimination of the low-energy part of the spectra that contains the unwanted components such as electronic noise, scattering etc. without affecting the energy resolution. In other words, low-energy part that contains the unwanted components due to electronic noise, scattering etc. discrimination using fast and slow signals in different spectrometers was performed through the used method.

Obtained experimental results showed that the second set-up gives better results in comparison with the first one. Although the first set-up has the capability of discrimination of the low-energy part of the energy spectrum, the $662 \mathrm{keV}$ peak (photo peak) of ${ }^{137} \mathrm{Cs}$ is not sharp compared to the spectrum from the second set-up. As can be seen in figure $2 \mathrm{~b}$, this conclusion is supported because of the high resolving time of the first set-up. 
It has been noticed that the result of second set-up obtained here was compatible with that of the previous work [13] containing different spectrometer components and their settings. In the study, the fall time criterion was employed using the pulse shape analysis observing the change in the fall times of the gamma ray pulses for Compton suppression of ${ }^{60} \mathrm{Co}$ and ${ }^{137} \mathrm{Cs}$. The PSD method using the rise times of the leading edges of the gamma ray pulses was implemented here for the elimination of unwanted parts of the spectrum differently from that work.

Since the discrimination of the low-energy part of the energy spectrum eliminates the effects originating from this part (such as electronic noise, background, scattering etc.), the method and the set-ups presented here will contribute to the acquisition of the neat energy spectrum.

\section{CONCLUSIONS}

By means of the PSD method, it can be possible to discriminate the unwanted signals from the energy spectrum partially or completely. Two different spectrometers were tested for this purpose. It was determined that these two spectrometers could discriminate the unwanted signals especially in the lowenergy region. It was revealed from the present study that a clearer gamma energy spectrum would be possible by the help of the PSD timing method used in these two spectrometers. The introduced spectrometers showed that they could be used for this purpose.

Consequently, two set-ups were suggested for partial or complete discrimination of the low-energy part of the gamma spectrum through the PSD method. It was shown from the study that the PSD method can successfully be used for the elimination of the unwanted parts of a gamma spectrum.

\section{ACKNOWLEDGMENTS}

This work was supported by TUBITAK, the Scientific and Technical Research Council of TURKEY under Projects No. 197T087, 104T379 and by EBILTEM, Center of Science and Technology, Ege University under Project No. 99 BIL 001.

\section{REFERENCES}

[1] Maas, A.J.H., Klein, S.S., Simons, D.P.L., de Voigt, M.J.A. (1996). Recoil selection by pulse shape discrimination in elastic recoil detection analysis with $\alpha$-particles. Nuclear Instruments and Methods in Physics Research B, 118(1-4), 268-273.

[2] Leo, W.R. (1994). Techniques for Nuclear and Particle Physics Experiment, Springer-Verlag, Berlin.

[3] Al-Haddad, M.N., Lin, C., Miller, W.H., Berliner, R.R. (1994). Development and testing of a flash analog-to-digital converter based system for pulse shape discrimination of nuclear radiation pulses. IEEE Transactions on Nuclear Science, 41(5), 1765-1769.

[4] Heistek, L.J., Zwan, L. van der, (1970). Pulse shape discrimination with a comparator circuit. Nuclear Instruments and Methods in Physics Research, 80(2), 213-216.

[5] Alexander, T.K., Goulding, F.S. (1961). An amplitude-insensitive system that distinguishes pulses of different shapes. Nuclear Instruments and Methods in Physics Research, 13, 244-246.

[6] Knoll, G.F. (2010). Radiation Detection and Measurement, Wiley, New York.

[7] Ruch, M.L., Flaska, M., Pozzi, S.A. (2015). Pulse shape discrimination performance of stilbene coupled to low-noise silicon photomultipliers. Nuclear Instruments and Methods in Physics Research A, 793, 1-5.

[8] Yanagida, T., Watanabe, K., Okada, G., Kawaguchi, N. (2019). Neutron and gamma-ray pulse shape discrimination of $\mathrm{LiAlO}_{2}$ and $\mathrm{LiGaO}_{2}$. Nuclear Instruments and Methods in Physics Research A, 919, 64-67.

[9] Doucet, E., Brown, T., Chowdhury, P., Lister, C.J., Morse, C., Bender, P.C., Rogers, A.M. (2020). Machine learning $\mathrm{n} / \gamma$ discrimination in CLYC scintillators. Nuclear Instruments and Methods in Physics Research A, 954, 161201. 
[10] Senoville, M., Delaunay, F., Parlog, M., Achouri, N.L., Orr, N.A. (2020). Neutron- $\gamma$ discrimination with organic scintillators: Intrinsic pulse shape and light yield contributions. Nuclear Instruments and Methods in Physics Research A, 971, 164080.

[11] Recker, M.C., Cazalas, E.J., McClory, J.W. (2020). Pulse shape discrimination with a low-cost digitizer using commercial off-the-shelf components. Nuclear Instruments and Methods in Physics Research A, 954, 161479.

[12] Sugiyama, Y., Ahn, J.K., Banno, S., Beckford, B., Campbell, M., Chen, S.H., Comfort, J., Duh, Y.T., Hineno, T., Hsiung, Y.B., Hutcheson, M., Iwai, E., Kamiji, I., Kawasaki, N., Kim, E.J., Kim, J.L., Kim, Y.J., Ko, J.W., Komatsubara, T.K., Kurilin, A.S., Lee, J.W., Lee, S.K., Lim, G.Y., Lin, C., Lin, Q., Luo, Y., Ma, J., Maeda, Y., Masuda, T., Matsumura, T., Mcfarland, D., Murayama, R., Naito, D., Nakagiri, K., Nakaya, Y., Nanjo, H., Nomura, T., Odani, Y., Redeker, J.C., Ri, Y.D., Sasao, N., Sato, K., Seki, S., Shimogawa, T., Shinkawa, T., Shinohara, S., Shiomi, K., Su, S., Suzuki, S., Tajima, Y., Takahashi, G., Takashima, Y., Tecchio, M., Togawa, M., Tung, Y.C., Wah, Y.W., Watanabe, H., Whallon, N., Woo, J.K., Xu, J., Yamanaka, T., Yanagida, Y., Yoshida, H.Y., Yoshimoto, H. (2021). Pulse shape discrimination of photons and neutrons in the energy range of 0.1 - $2 \mathrm{GeV}$ with the KOTO un-doped CsI calorimeter. Nuclear Instruments and Methods in Physics Research A, 987, 164825.

[13] Selvi, S., Celiktas, C. (2007). Compton suppression through rise-time analysis. Applied Radiation and Isotopes, 65, 1265-1268. 